\title{
EXCIPIENTS AND THEIR ROLE IN ABSORPTION: INFLUENCING BIOAVAILABILITY OF CYCLOSPORINE BY TRIGLYCERIDES AND POLYGLYCEROL ESTERS
}

\author{
Tomas Andrysek
}

\author{
Generic Pharmaceutical RD Department, IVAX Pharmaceuticals Ltd., Ostravsk 29, Opava 9, Czech Republic \\ e-mail:tomas_andrysek@ivax-cz.com
}

Received: September 15, 2006; Accepted: November 15, 2006

Key words: Absorption/Cyclosporine/Polyglycerol esters/Triacylglycerols/Equoral/Intraduodenal application

\begin{abstract}
Aims: The goal of the experiment was to verify bioavailability of immunosuppressive drug cyclosporine after oral administration, to determine, in particular, effect of triglycerides and polyglycerol esters on bioavailability of this lipophilic, poorly soluble drug.

Methods: 1) Absorption of cyclosporine A from soya oil and polyglycerol-3-oleate was tested after intra-duodenal application to rats. This method enable to administer dispersion directly to the site of absorption and avoid problems with potential precipitation of active substances in the stomach. Samples were pre-dispersed in water and diluted on concentration $1 \mathrm{mg} / \mathrm{ml}$ prior administration. In defined time intervals a blood sample was taken in a volume of $0.5 \mathrm{ml}$ from the cannuled carotid. Blood was sampled in time 5, 15, 30, 45, 60, 90, 120, 180 and 240 minutes after application. For the comparison, Equoral ${ }^{\circledR}$ oral solution diluted and pre-dispersed in the same manner was used. 2) $100 \mathrm{mg}$ of cyclosporin in form of $1 \%$ dispersion in water was administered orally to dogs under fasting condition. The blood level of cyclosporine was evaluated from samples taken in time $0.5,1,1.5,2,2.5,3,5,8,12$ and 24 hours following application. Formulations containing different ratio of polyglycerol esters / olive oil were compared.

Results: The experiments conducted indicate that cyclosporine is $19 \times$ more available from polyglycerol-3-oleate than from soya oil. When applying cyclosporine in polyglycerol-3-oleate the average maximum blood level is $10 \mathrm{x}$ higher then in application of cyclosporine in oil. If polyglycerols are fully substituted with plant oils in the formula observed, its pharmacokinetic parameters decrease to $1 / 10$ of the initial values.

Conclusions: The right selection of a type of excipient accompanying cyclosporine affects significantly cyclosporine availability and thus its efficiency.
\end{abstract}

\section{INTRODUCTION}

Cyclosporine $\mathrm{A}$ is a potent immunosuppressive drug which is used for more then 20 years in clinical practice. In 1983 cyclosporine A in form of oral solution and injection was approved by FDA under the brand name Sandimmune $^{\circledR}$, Sandoz Ltd. Later on, in 1990, it was followed by soft gelatine capsules in USA. At the same time first generic version of cyclosporine A containing oral solution was approved in Czechoslovakia under the brand name Consupren ${ }^{\circledR}$, Galena s.p.. Nowadays, second generation of cyclosporine A containing product is widely used and differs from first generation. The main difference between first and second generation of products, besides improved bioavailability of cyclosporine A, is presence of triacylglycerols (also known as triglycerides or natural, mainly vegetable oils). Whilst all first generation products have used as part of vehicle, in which cyclosporine A was dissolved, triacylglycerols, all second generation products use mixture of amphiphilic compounds with different level of lipophilicity, surface activity, which are easily dispersible in water.

In the past, the only reason for increased absorption of cyclosporine A from second generation product was seen in reduction of particle size rising in contact of product with aqueous milieu prior or after application ${ }^{1,2}$.

Early findings were later on in contrast with facts that also crude types of nascent dispersions allow to obtain the same bioavailability of cyclosporine $\mathrm{A}$ in blood as with very fine microemulsion ${ }^{3,4,5}$.

As the first, physical aspect of novel highly available formulation was investigated gel formation upon contact with aqueous milieu ${ }^{6,7}$, which was associate with phase transition, when liquid crystalline phase is rising. In spite the fact that liquid crystalline phase has bio-adhesive character and mechanistic interpretation is thus offered, also character of individual excipients and theirs potential to affect bioavailability was considered ${ }^{8,9}$.

Equoral $^{\circledR}$, the second generation of product containing cyclosporin A developed in Opava contain besides active substance, ethanol and polyoxyethylated surfactant also polyglycerol esters. Because the role of ethanol and polyoxyethylated surfactant is well know and well described ${ }^{10}$, in current study we have focused on the role of polyglycerol esters. Because polyglycerol esters are amphiphilic molecules with higher affinity to oil and thus they are called lipophilic surfactants, we intended to compare theirs effect on bioavailability with true lipophilic, pharmaceuticaly acceptable oils. 


\section{MATERIAL AND METHODS}

The samples containing cyclosporine were prepared in the Laboratory of Dosage Forms Formulation, IVAX Pharmaceuticals, s.r.o. Opava. The biological experiments involving rats were carried out in the Department of Pharmacology and Toxicology of the Pharmaceutical Faculty of UK Hradec Králové. The biological experiments involving dogs were carried out by BIOTEST s.r.o. in Konárovice. Analytical evaluation of samples was carried out in laboratories of the Nuclear Medicine in FNsP Ostrava.

Preparation of Samples: The following starting materials were used, hereinafter they are specified by a name (quality) and manufacturer's name: cyclosporine A ( Ph. Eur), IVAX Pharmaceuticals (CZ); ethanol anhydrous (Ph.Eur), Moravian Distillery Kojetín (ZC); soya oil refined EP (Ph.Eur), Karlshamns AB (Sweden); olive oil refined (PhEur.), G.Hess (Germany); polyglycerol-3oleate (E475), Danisco (Denmark), Polyglycerol-10-oleate (INS:475), Abitec (USA), hydrogen-ricino-macrogol 2000 (BASF), Germany.

\section{Sample I (also refered as $\mathrm{Vz} \mathrm{I}$ );}

$100 \mathrm{mg}$ of cyclosporine / $\mathrm{ml}$ :

$10.0 \mathrm{~g}$ of cyclosporine $\mathrm{A}$ is dissolved in $12 \mathrm{ml}$ of anhydrous ethanol at the temperature of $50^{\circ} \mathrm{C}$, than soya oil is added to a total volume of $100 \mathrm{ml}$ in a flask.

\section{Sample II (also refered as Vz II);}

$100 \mathrm{mg}$ of cyclosporine / $\mathrm{ml}$ :

$10.0 \mathrm{~g}$ of cyclosporine A is dissolved in $12 \mathrm{ml}$ of anhydrous ethanol at the temperature of $50{ }^{\circ} \mathrm{C}$, than polyglycerol-3-oleate is added to a total volume of $100 \mathrm{ml}$ in a flask.

Equoral oral solution, $100 \mathrm{mg} / \mathrm{ml}$, batch: 4R310001;

IVAX Pharmaceuticals s.r.o. (CZ):

Commercially available product containing, in addition to cyclosporine, anhydrous ethanol, polyglycerol-3-oleate, polyglycerol-10-oleate and hydrogen-ricino-macrogol 2000.

\section{EQ-SUBST-P3, $100 \mathrm{mg} / \mathrm{ml}$;;}

A sample prepared in laboratory, of the composition based on that of Equoral oral solution in which the component polyglycerol-3-oleate is replaced with $31 \% \mathrm{w} / \mathrm{w}$ of olive oil.

\section{EQ-SUBST-P10, 100 mg/ml,;}

A sample prepared in laboratory, of the composition based on that of Equoral oral solution in which the component polyglycerol-10-oleate is replaced with replaced with $19 \%$ w/w of olive oil.

\section{EQ-SUBST-P3+10, $100 \mathrm{mg} / \mathrm{ml}$;}

A sample prepared in laboratory, of the composition based on that of Equoral oral solution in which the components polyglycerol-3-oleate and polyglycerol-10-oleate are replaced with replaced with $50 \% \mathrm{w} / \mathrm{w}$ of olive oil.

\section{Animals:}

Rat Males (Wistar), 9-11 weeks old, of a weight of 250 to $300 \mathrm{~g}$, bought from the Biotest s.r.o. Konárovice breed, were used for the study of intraduodenal applica- tion. The animals were divided into groups by 5 and kept in standard conditions (12-hour light regime at $22^{\circ} \mathrm{C}$; pellet feeding and water ad libitum) for minimum a week before the start of the study so that adaptation of them was ensured. 18 hours before the experiment the pellet feed was taken from the rats while they still had free access to water. One formula containing cyclosporine was applied to each group of rats.

Dog Males (Beagle) of a weight of $10-15 \mathrm{~kg}$ coming from the internal breeding of Biotest, s.r. o., Konárovice were used for the study of cyclosporine availability following peroral administration. The group of animals intended for the experiment was kept under standard conditions (12-hour light regime at $15-21^{\circ} \mathrm{C}$; pellet feeding and water ad libitum). 18 hours before the experiment the pellet feed was taken from the dogs while they still had free access to water. In each case one formula containing cyclosporine was applied as a single dose. Cyclosporine levels were observed for a period of 24 hours after the application. Then the dogs were put back to the breed and they have at least a 14-day rest (washout) period.

\section{Intraduodenal Application (Rats):}

The rats were anaesthetized with pentobarbital (Nembutal ${ }^{\circledR}$, Abbott Laboratories, North Chicago, IL, USA), in a dose of $40 \mathrm{mg} / \mathrm{kg}$, administered via intravenous application into the tail vein. The anaesthetized rats were put on a surgical table and cannuled into a carotid with a PVC cannula filled with heparin; the cannula was fixed firmly and the point of cannula incision was kept wetted with saline solution. The abdominal cavity was opened and the distal part of duodenum was exposed.

The tested samples, pre-dispersed with water, at concentration of $1 \mathrm{mg} / \mathrm{ml}$ were applied in a dose of $5 \mathrm{mg} / \mathrm{kg}$ to the exposed distal duodenum via a micro-needle in time 0 (zero). After the application the duodenum was closed with tissue glue Histoacryl ${ }^{\circledR}$, abdominal cavity was closed with 3 stitches and the site of intervention was kept wet with saline solution.

In defined time intervals a blood sample was taken in a volume of $0.5 \mathrm{ml}$ from the cannuled carotid. Blood was sampled in time $5,15,30,45,60,90,120,180$ and 240 minutes after application of the product to TAPVAL tubes containing anticoagulant. Following each sampling the cannula was washed with heparinized saline solution. The blood samples were kept frozen at $-28^{\circ} \mathrm{C}$ until they were analyzed. After the last sampling the animals were killed via overdosing of anaesthetics.

All experiments were approved by the Ethics Committee of the Pharmaceutical Faculty of UK, Hradec Králové, and were conducted in accordance with directive "Guide for the Care and Use of Laboratory Animals“, from 1996, and by the European Convention for the Protection of Vertebrate Animals Used for Experimental and Other Purposes, Strasbourg, 1986.

\section{Peroral administration (dogs):}

The fasting dogs were administered a single dose of $100 \mathrm{mg}$ of cyclosporine in a form of $1 \%$ dispersion in water (i.e. $1 \mathrm{ml}$ of drug dispersed in $99 \mathrm{ml}$ of water) through 
a peroral tube. In defined time intervals 2 blood samples were taken from the antecubital vein, each in a volume of $1.0 \mathrm{ml}$. The blood was taken in time $0.5,1,1.5,2,2.5,3$, $5,8,12$ and 24 hours following application of the product to TAPVAL tubes containing anticoagulant. It was frozen till the time of analysis at $-18 \pm 2{ }^{\circ} \mathrm{C}$.

Analytics: Concentration of cyclosporine in blood samples was analyzed via a validated method in the laboratory of Nuclear Medicine Clinic of FNsP Ostrava using specific radioimmune analysis (DiaSorin RIA, Specific).

\section{RESULTS AND DISCUSSION}

Sample I, Sample II and Equoral were administered intraduodenaly. In all three cases measurable levels of cyclosporine were found following administration. However, the results imply that the active ingredient (cyclosporine) is absorbed differently depending on a type of formula. It is thus clear that various excipients affect differently cyclosporine bioavailability and have thus different influence on its potential effect.

The difference is the most clearly visible in Figure 1. There is shown comparison of cyclosporine average levels in rat blood after single-dose intraduodenal application (ID application) of dose $5 \mathrm{mg} / \mathrm{kg}$. The diagram clearly demonstrates a dramatic difference in polyglycerols and triglycerides characteristics. While the sample Sample II, which contains cyclosporine dissolved in polyglycerol3-oleate, shows Cmax around $1022 \mathrm{ng} / \mathrm{ml}$, the sample Sample I that contains cyclosporine dissolved in soya oil shows c.10× lower Cmax, around $110 \mathrm{ng} / \mathrm{ml}$. If we compare the total bioavailability expressed as $\mathrm{AUC}_{0 \text {-inf, }}$

Table 1. Average values of pharmacokinetic parameters after intra-duodenal application on rats.

\begin{tabular}{|l|c|c|c|c|c|c|c|c|c|}
\hline & $\begin{array}{c}\text { Equoral sol. } \\
\text { (average) }\end{array}$ & SD & RSD \% & $\begin{array}{c}\text { Sample I } \\
\text { (average) }\end{array}$ & SD & RSD \% & $\begin{array}{c}\text { Sample II } \\
\text { (average) }\end{array}$ & SD & $\begin{array}{c}\text { RSD } \\
\%\end{array}$ \\
\hline AUC $_{0-240}$ & 313553.0 & 190251.53 & 61 & 10777.8 & 6708.39 & 62 & 192826.6 & 183356.40 & 95 \\
\hline Cmax & 1603.4 & 888.74 & 55 & 110.1 & 130.80 & 119 & 1022.4 & 927.61 & 91 \\
\hline Tmax & 102.0 & 26.83 & 26 & 150.0 & 90.00 & 60 & 151.0 & 57.49 & 38 \\
\hline Kel & 0.0051 & 0.00 & 0 & 0.0051 & 0.00 & 0 & 0.0051 & 0.00 & 0 \\
\hline AUC $_{\text {t-inf }}$ & 250378.2 & 136858.68 & 55 & 8327.6 & 6699.68 & 80 & 175756.7 & 162417.85 & 92 \\
\hline AUC $_{0-\text { inf }}$ & 563931.2 & 326915.31 & 58 & 19105.4 & 11352.07 & 59 & 368583.3 & 345641.28 & 94 \\
\hline
\end{tabular}

$\mathrm{SD}=$ standard deviation; $\mathrm{RSD}=$ relative standard deviation in $\% ; \mathrm{AUC}_{0-240}=$ an area under the curve in time $0-240$ min; $\mathrm{Cmax}=$ peak concentration observed after administration; $\mathrm{Tmax}=$ calculated mean time, when Cmax was observed; $\mathrm{Kel}=$ calculated elimination constant; $\mathrm{AUC}_{\mathrm{t} \text {-inf }}=$ an area under the curve approximated from 24 time point to infinity; $\mathrm{AUC}_{0 \text {-inf }}=$ an area under the curve approximated from 0 time point to infinity; $\mathrm{Cl}=$ clearance

Table 2. Average values of pharmacokinetic parameters after peroral administration on dogs.

\begin{tabular}{|l|c|c|c|c|c|c|c|c|c|c|c|c|}
\hline & \multicolumn{3}{|c|}{ Equoral sol. } & \multicolumn{3}{c|}{ EQ-SUBST-P3 } & \multicolumn{3}{c|}{ EQ-SUBST-P10 } & \multicolumn{3}{c|}{ EQ-SUBST-P3+P10 } \\
\hline & Average & SD & RSD $\%$ & Aver. & SD & RSD $\%$ & Aver. & SD & RSD\% & Aver. & SD & RSD\% \\
\hline AUC $_{\mathbf{0 - 2 4 h}}$ & 8801.2 & 2378.97 & 27.0 & 5674.1 & 2281.71 & 40.21 & 7932.3 & 1354.90 & 17.08 & 905.0 & 144.14 & 15.9 \\
\hline $\mathbf{C}_{\text {max }}$ & 1359.1 & 421.39 & 31.0 & 979.4 & 572.90 & 58.49 & 1276.4 & 282.41 & 22.13 & 130.8 & 41.78 & 31.9 \\
\hline $\mathbf{T}_{\text {max }}$ & 1.3 & 0.45 & 34.4 & 1.5 & 0.35 & 23.57 & 1.7 & 0.57 & 33.53 & 1.5 & 0.61 & 40.8 \\
\hline Kel & -0.0436 & 0.01 & 17.3 & -0.0489 & 0.01 & 12.66 & -0.0433 & 0.00 & 8.36 & -0.0302 & 0.00 & 6.4 \\
\hline AUC $_{\text {t-inf }}$ & 2357.9 & 1216.20 & 51.6 & 1143.0 & 602.16 & 52.68 & 2235.1 & 702.27 & 31.42 & 554.2 & 100.16 & 18.1 \\
\hline AUC $_{\text {0-inf }}$ & 11159.1 & 3276.24 & 29.4 & 6817.1 & 2797.83 & 41.04 & 10167.4 & 2045.41 & 20.12 & 1459.2 & 234.85 & 16.1 \\
\hline $\mathbf{C l}$ & 9.6 & 2.63 & 27.5 & 16.5 & 5.54 & 33.63 & 10.1 & 1.91 & 18.86 & 69.8 & 10.19 & 14.6 \\
\hline
\end{tabular}

$\mathrm{SD}=$ standard deviation; $\mathrm{RSD}=$ relative standard deviation in $\%$; $\mathrm{AUC}_{0-24}=$ an area under the curve in time 0-24 hrs; $\mathrm{Cmax}=$ peak concentration observed after administration; $\mathrm{Tmax}=$ calculated mean time, when Cmax was observed; $\mathrm{Kel}=$ calculated elimination constant; $\mathrm{AUC}_{\mathrm{t} \text {-inf }}=$ an area under the curve approximated from 24 time point to infinity; $\mathrm{AUC}_{0 \text {-inf }}=$ an area under the curve approximated from 0 time point to infinity; $\mathrm{Cl}=$ clearance 
Comparison of CyA Bioavailability - ID Application (Rats)

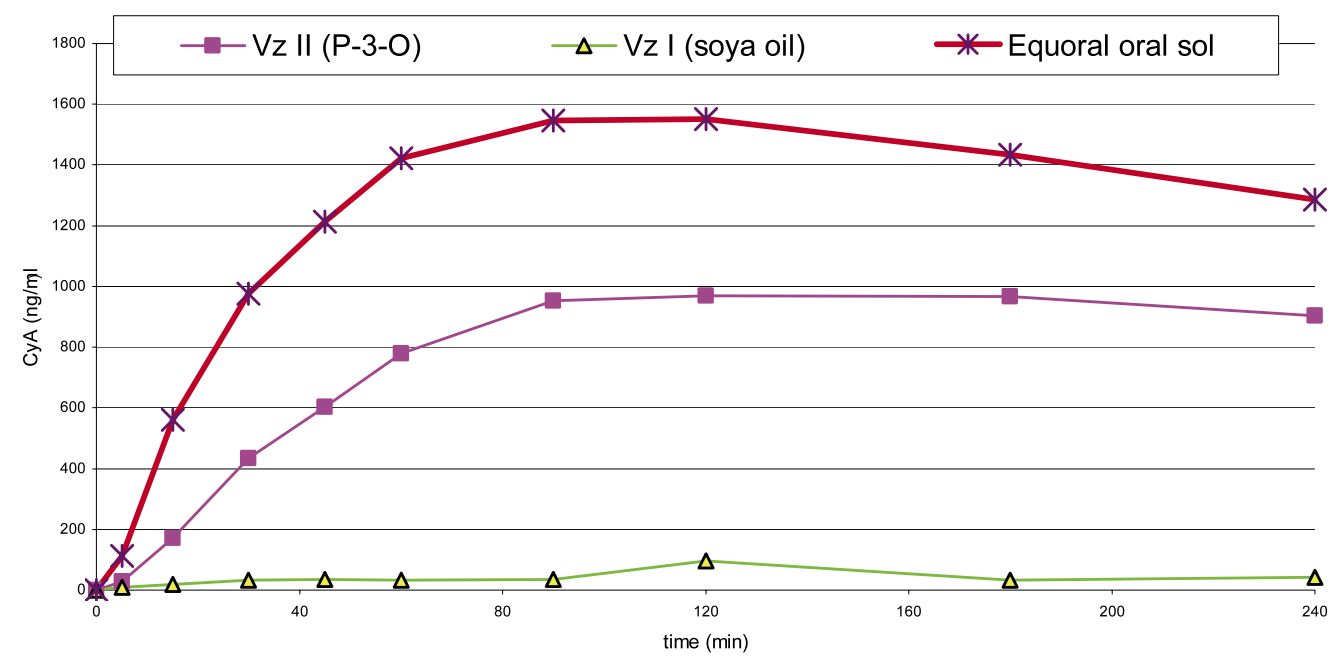

Vz I = Sample I: $10 \% \mathrm{w} / \mathrm{v}$ solution of cyclosporine A in soybean oil with standard content of ethanol; Vz II = Sample II: $10 \%$ w/v solution of cyclosporine A in polyglycerol-3-oleate with standard content of ethanol; Equoral oral solution: $10 \% \mathrm{w} / \mathrm{v}$ solution of cyclosporine A in mixture of polyglycerol esters, hydrogen-ricino-macrogol 2000 with standard content of ethanol

Fig. 1. Comparison of courses of cyclosporine average levels in rat blood after single-dose intraduodenal application of $5 \mathrm{mg} / \mathrm{kg}$ of cyclosporine A pre-dispersed in water in concentration $1 \mathrm{mg} / \mathrm{ml}$.

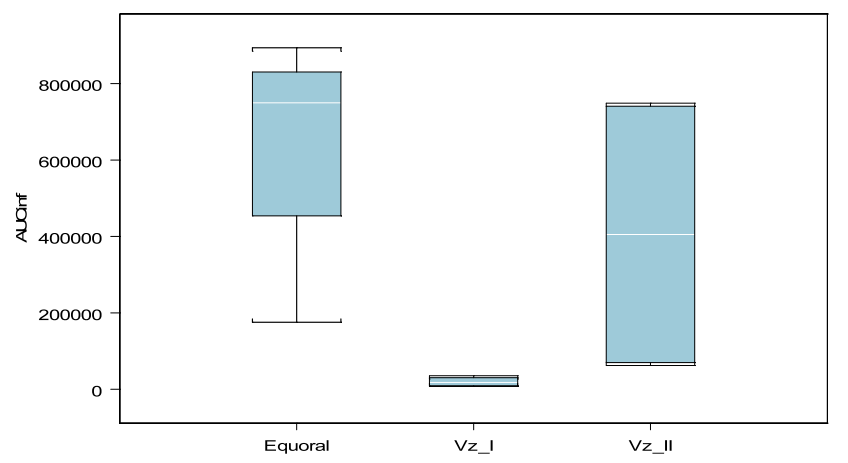

$\mathrm{Vz} \mathrm{I}=$ Sample I: $10 \% \mathrm{w} / \mathrm{v}$ solution of cyclosporine A in soybean oil with standard content of ethanol; $\mathrm{Vz} \mathrm{II} \mathrm{=} \mathrm{Sam-}$ ple II: $10 \% \mathrm{w} / \mathrm{v}$ solution of cyclosporine $\mathrm{A}$ in polyglycerol3-oleate with standard content of ethanol; Equoral oral solution: $10 \% \mathrm{w} / \mathrm{v}$ solution of cyclosporine $\mathrm{A}$ in mixture of polyglycerol esters, hydrogen-ricino-macrogol 2000 with standard content of ethanol; $\mathrm{AUC}_{\text {inf }}=$ an area under the curve approximated from 0 time point to infinity

Fig. 2. Box diagram illustrating comparison of mean values of bioavailability (expressed as an under-curve area) of individual formulas in rats.

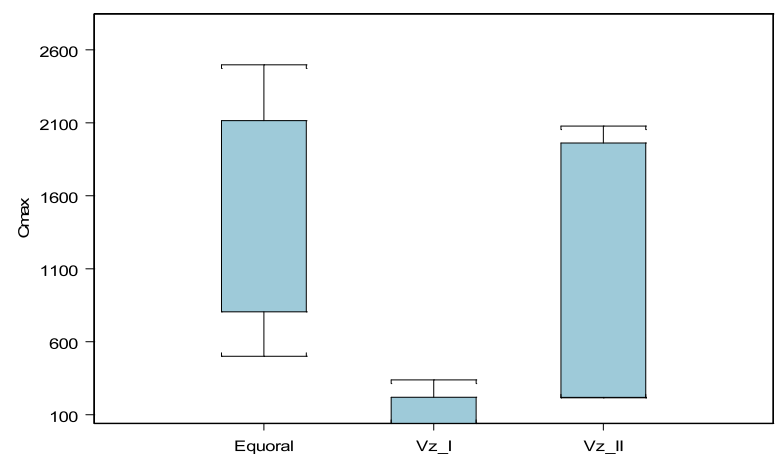

$\mathrm{Vz} I=$ Sample I: $10 \% \mathrm{w} / \mathrm{v}$ solution of cyclosporine A in soybean oil with standard content of ethanol; Vz II = Sample II: $10 \% \mathrm{w} / \mathrm{v}$ solution of cyclosporine $\mathrm{A}$ in polyglycerol3-oleate with standard content of ethanol; Equoral oral solution: $10 \% \mathrm{w} / \mathrm{v}$ solution of cyclosporine $\mathrm{A}$ in mixture of polyglycerol esters, hydrogen-ricino-macrogol 2000 with standard content of ethanol; $\mathrm{Cmax}=$ peak concentration observed after administration

Fig. 3. Box diagram illustrating comparison of mean values of maximum concentrations of cyclosporine following intraduodenal application of individual formulas in rats. 


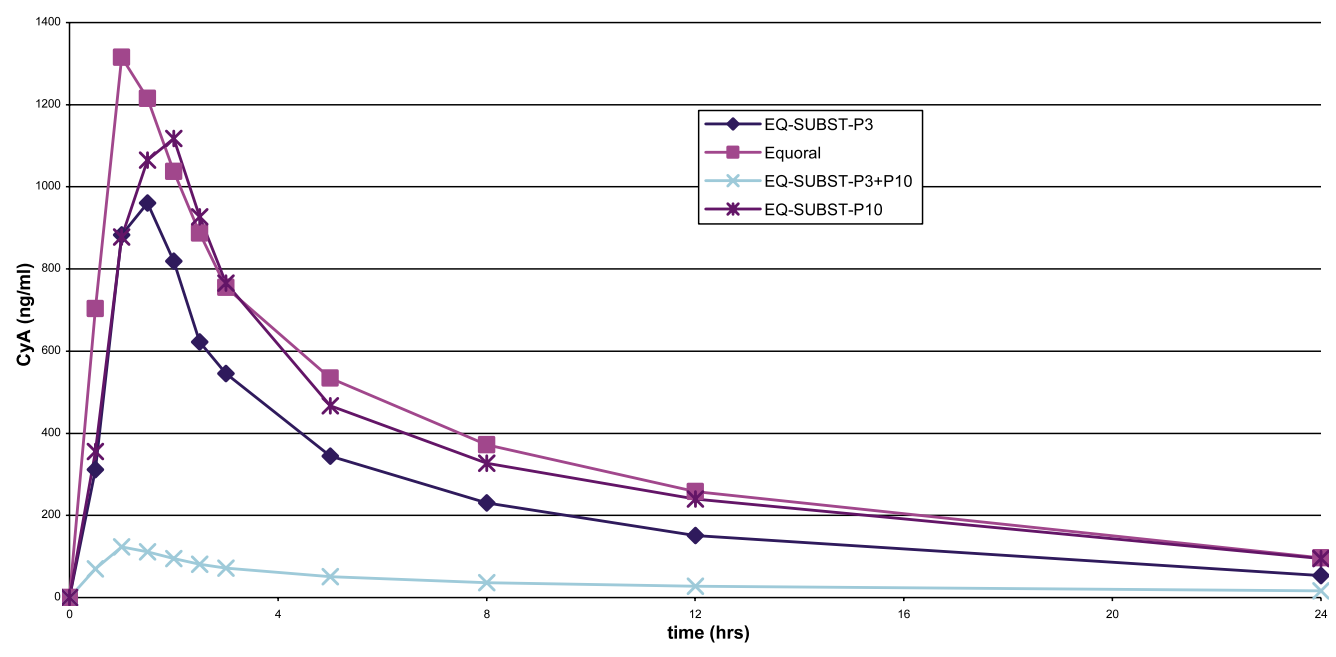

Equoral $=$ cyclosporin A containing formulation which do not contain triacylglycerols; EQSUBST-P-10 = equoral-like formulation where $2 / 5$ of total amount of polyglycerol esters were substituted by triglycerides; EQ-SUBST-P-3 = equoral-like formulation where $3 / 5$ of total amount of polyglycerol esters were substituted by triglycerides; EQ-SUBST-P-3+P-10 = equoral-like formulation all polyglycerol esters were substituted by triglycerides

Fig. 4. Comparison of courses of cyclosporine average levels in dog blood after single-dose oral administration of $100 \mathrm{mg}$ of cyclosporine $\mathrm{A}$ in form of $1 \%$ dispersion in water.

the difference which is in aid of the polyglycerol-3-oleatebased sample is even bigger. The total bioavailability of the sample Sample II is c. 19x higher than that of Sample I (see Table 1). In other words, cyclosporine bioavailability from triacylglycerol, represented by soya oil, is $19 \times$ lower than bioavailability of the same substance from polyglycerol-3-oleate.

Statistical comparison of values $\mathrm{AUC}_{\text {inf }}$ and $\mathrm{Cmax}$ of samples Sample I and Sample II confirmed statistically significant difference of both populations at the significance level of $p=0.05$ (Fig. 2).

This fact is most probably a result of several factors that influence bioavailability of active ingredients. Partly, it is a factor of solubility of active ingredient in the respective solvent. Then there is a factor of lipophilicity and surface activity. Cyclosporine is c. $5-7 \times$ more soluble in polyglycerol-3-oleate than in triacylglycerols in general. However, this fact does not correspond to $19 \times$ higher bioavailability. There is thus another fact playing an important role - polyglycerol-3-oleate being a surfactant.

Making a narrow classification we can consider polyglycerol-3-oleate a substance with affinity to oils (i.e. a lipophilic substance), however, its intrinsic character is strictly amphiphilic - with affinity to both oil and water phase. Within intentions of the amphiphillic group of substances its character can be classified as more lipophilic than that of e.g. polyglycerol-10-oleate or hydrogen-ricinomacrogol 2000 that are used in Equoral as well. Such amphiphility results in good dispersability of the substance and tendency to remain in dispersed status (dispersion stability). The effect has significantly positive influence on bioavailability of substances dissolved in polyglycerol3-oleate.

By contrast, soya oil has no ability of spontaneous dispersability in water and thus it is full-lipophilic substance. If we apply a force dispersing soya oil in water the oil significantly tends, on the contrary, to separate from water. This effect, undesirable from drug-absorption point of view, has a negative effect on bioavailability of substances based on triacylglycerols. In terms of these different characteristics it is impossible to consider them functional equivalents in regard of cyclosporine dosage forms. From that point of view, the use of polyglycerol esters is more favourable than the use of triacylglycerols.

In order to compare bioavailability of Sample I and Sample II on rats final dosage form - Equoral oral solution -was also applied in this case. This dosage form which also contains other excipients that optimize both product stability and its effect shows even $1.5 \times$ higher bioavailability than Sample II does. Figure 2 and 3 thus demonstrate that average mean values of Sample II are not significantly different from Equoral values.

The negative effect of triacylglycerols on bioavailability of cyclosporine is also illustrated by the experiment summarized in Figure 4. In this case, dispersions containing cyclosporine derived from medicinal product Equoral were administered per os. However, the dispersions were different in the sense that polyglycerol esters contained in Equoral were replaced with triacylglycerol, precisely olive oil. Equoral contains two types of polyglycerol esters: c. $3 / 5$ of polyglycerol-3-oleate which is more lipophilic and c. $2 / 5$ of polyglycerol-10-oleate which is more hydrophilic. In 
Effect of Olive Oil Ratio in Formula on Bioavailability Parameters

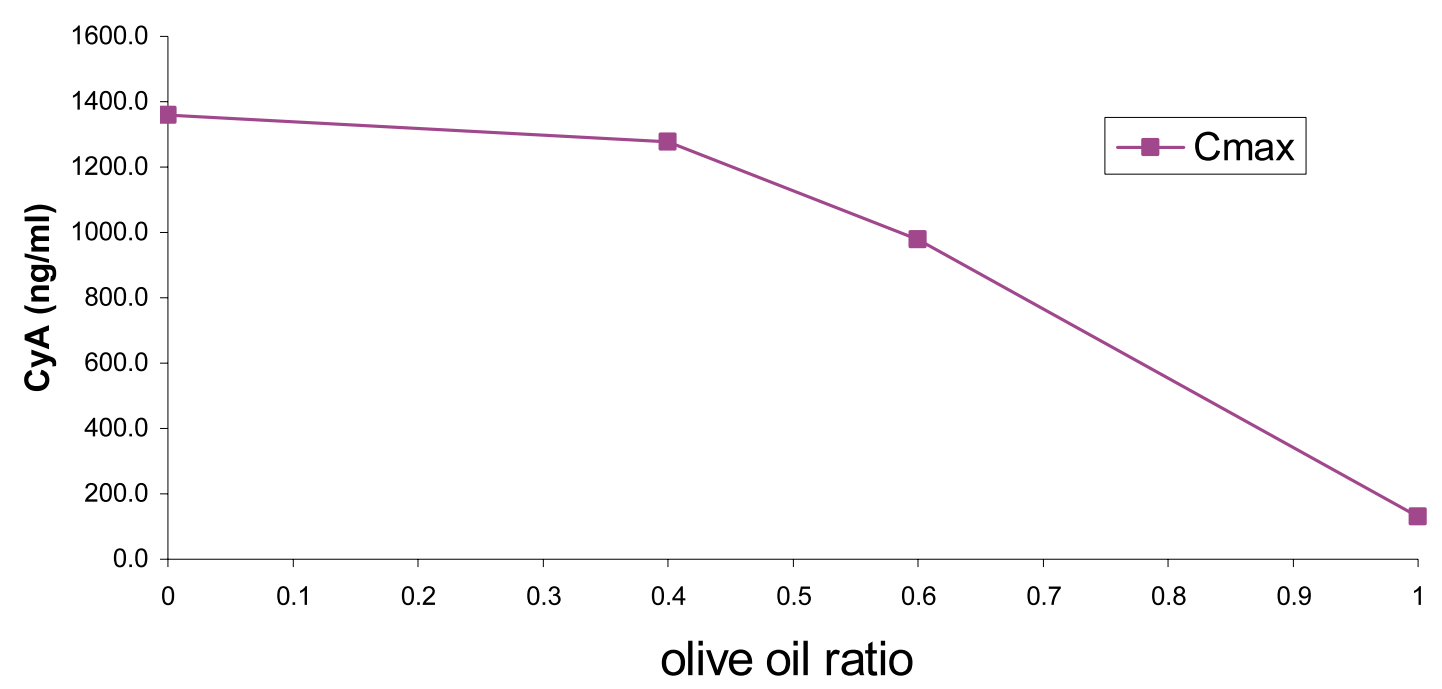

$\mathrm{Cmax}=$ peak concentration observed after administration; olive oil ratio $=$ relative polyglycerol ester/olive oil ratio in tested formulation

Fig. 5. Effect of polyglycerol substitution with triacylglycerols on Cmax. after single-dose oral administration of $100 \mathrm{mg}$ of cyclosporine $\mathrm{A}$ in form of $1 \%$ dispersion in water on dogs.

Effect of Olive Oil Ratio in Formula on Bioavailability Parameters

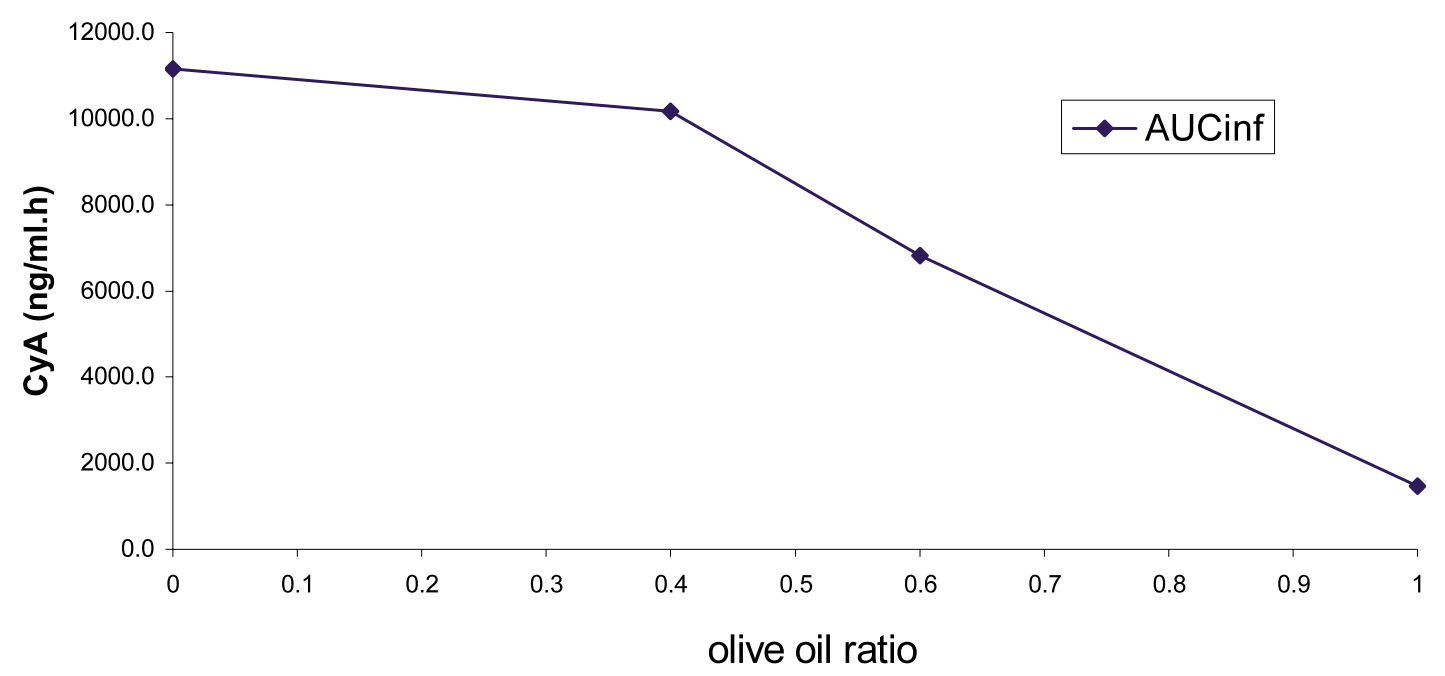

$\mathrm{AUC}_{\text {inf }}=$ an area under the curve approximated from 0 time point to infinity; olive oil ratio = relative polyglycerol ester/olive oil ratio in tested formulation

Fig. 6. Effect of polyglycerol substitution with triacylglycerols on $\mathrm{AUC}_{\text {inf }}$ after single-dose oral administration of $100 \mathrm{mg}$ of cyclosporine $\mathrm{A}$ in form of $1 \%$ dispersion in water on dogs.

the experiment, polyglycerol-10-oleate was replaced with olive oil at first while quantitative aspects of composition in sample EQ-SUBST-P10 were kept. Then polyglycerol3-oleate was substituted with olive oil in sample EQSUBST-P3 and finally, both polyglycerols were substituted in sample EQ-SUBST-P3+10. In other words, the sample contained $2 / 5,3 / 5$ and $5 / 5$ of polyglycerol esters substituted in their composition with olive oil. In proportion to the substitution it can be seen decline in cyclosporine blood levels in Table 2. The Table demonstrates that if polyglycerol esters are fully replaced with olive oil, $\mathrm{AUC}_{\mathrm{inf}}$ of the respective sample decreases to c. $1 / 10$ of the value of the sample with polyglycerol esters. Figure 5 and 6 enable derivation of exponential dependence of decrease in observed parameters $\mathrm{Cmax}$ and $\mathrm{AUC}_{\text {inf }}$ on increasing ratio of triacylglycerols. The dependence can be described 
as polynom of second degree with very good correlation coefficient ranging 0.98-0.99.

The experiment on dogs is all the more valuable that it ran in a cross regime - all compositions were applied to identical animals after the end of the wash-out period. Also this experiment proves the fact that if polyglycerol esters are substituted with triacylglycerols in a controlled experiment, significantly different characteristics of bioavailability of cyclosporine-like lipophilic substances are observed.

\section{CONCLUSION}

Selection of a type of excipient accompanying cyclosporine affects significantly cyclosporine availability and thus its efficiency.

Polyglycerol esters represent safe and well-tolerable surfactants, which can be used in formulation without negative influence on bioavailability of co-administered drug. They are used for more then 60 years in alimentary industry ${ }^{11}$. After administration fatty acids are hydrolyzed in GI tract from polyglycerol backbone and are available for absorption and utilization as would occur with natural dietary fatty acids consumed in diet. Shorter polyglycerols can be also absorbed and unchanged excreted by urine, longer polyglycerols are not absorbed and are excreted to carcass $^{12}$. In clinical study on healthy volunteers $10 \mathrm{~g} /$ day polyglycerol esters in diet did not affect normal fat digestion and absorption ${ }^{13}$.

The experiments conducted indicate that cyclosporine is $19 \times$ more available from polyglycerol-3-oleate than from soya oil. When applying cyclosporine in polyglycerol-3oleate the average maximum blood level is $10 \times$ higher then in application of cyclosporine in oil. If polyglycerols are fully substituted with plant oils in the formula observed, its pharmacokinetic parameters decrease to $1 / 10$ of the formula without plant oil.

\section{REFERENCES}

1. Tarr BD, Yalkowsky SH. Enhanced intestinal absorption of cyclosporine in rats through the reduction of emulsion droplet size. Pharmaceutical Research, 1989, 6, 1:40-3.

2. Hutchinson KG, MacGregor K, Embleton JK, Seager H, Solomon $\mathrm{LJ}$ and Pouton CW. Optimizing the gastro-intestinal absorbtionof hydrophobic drugs using micro-emulsions and other liquid formulations. In: Couvreur P, Duchene D, Kalles I, editors. Formulation of poorly available drugs for oral administration. Paris: Editions de Santé; 1995; $121-5$.

3. Andrýsek T, Vrána A, Stuchlík M: The effect of particle size on bioavailability for cyclosporine preparations based on the formation of submicron dispersion. Eur J Pharm Sci 1998; 8; Suppl. 1:S 77

4. Andrýsek T, Pisárčik M. Bioavailability versus physical properties in cyclosporin-containing drug delivery systems. In: Proceedings from Symposium on „Lipid and surfactant dispersed system“. 1999 26-28 September; Moscow; p. 187-8.

5. Vrána A, Andrýsek T. The effect of particle size on bioavailability in cyclosporine preparations based on submicron dispersions. Biomed. Papers 2001, 145(2):9-15.

6. Andrýsek T, Uhrikova D, Son D, Funari SS, Balgavy P. Determination of supramolecular structure of EQUORAL ${ }^{\circledR}$, novel drug delivery system with cyclosporine. In: Proceedings $30^{\text {th }}$ Annual CRS Meeting, 2003 20-23 July; Glasgow, UK. Minneapolis: Controlled Release Society; 2003, 210.

7. Uhríková D, Andrýsek T, Funari SS, Balgavý P. Synchrotron radiation small- and wide- angle scattering study of dispergation of Equoral ${ }^{\circledR}$, a novel drug delivery system with cyclosporine A. Pharmazie 2004, 59:650-1.

8. Andrýsek T. The role of particle size distribution on bioavailability of cyclosporine: novel drug delivery system. Biomed. Papers 2001, 145:5-10.

9. Murdan S, Andrýsek T, Son D. Novel gels and their dispersions - oral drug delivery systems for ciclosporin. Int J Pharm 2005, 300: 113-24.

10. Andrýsek T. The study of self-dispersing liquid systems. (Disertation) Pharmaceutical Faculty of Charles University, Hradec Králové, Czech Republic, 2005.

11. Bodansky M, Herrmann CL and Campbell K. Utilization of polyglycerol esters. Biochem J 1938, 32(11):1938-42.

12. Michael WR and Coots RH. Metabolism of polyglycerol and polyglycerol esters. Toxicol Appl Pharmacol 1971, 20(3):334-45.

13. Wilson R and Smith M. Human studies on polyglycerol polyricinoleate (PGPR). Food Chem Toxicko 1998, 36(9-10):743-5. 